\title{
On water waves generated by gravity driven granular collapse
}

\author{
Wladimir Sarlin ${ }^{1, *}$, Cyprien Morize ${ }^{1, * *}$, Alban Sauret ${ }^{2}$, and Philippe Gondret ${ }^{1}$ \\ ${ }^{1}$ Université Paris-Saclay, CNRS, Laboratoire FAST, F-91405 Orsay, France \\ ${ }^{2}$ University of California, Santa Barbara, Department of Mechanical Engineering, USA
}

\begin{abstract}
Tsunami-like waves can arise from large-scale geological events such as the collapse of a mountain flank or a volcano into a lake or an ocean. We here mimic this situation at the laboratory scale. The landslide is modeled by the sudden release of a rectangular granular column, which impacts a still water layer and generates a wave. Through experiments varying both the column dimensions and the initial water depth, three regimes of nonlinear waves of different shapes are reported. The amplitude and width of the generated waves are governed by a local Froude number based on the velocity of the granular front at the water surface.
\end{abstract}

\section{Introduction}

A tsunami wave can be generated by the collapse of the flank of a mountain or a volcano into a lake or ocean. A well-known example is the case of Lituya Bay (Alaska, USA) in 1958 [1], when a rockslide volume of $30 \times 10^{6} \mathrm{~m}^{3}$ fell into water and produced a large wave with a recorded runup of $524 \mathrm{~m}$ along the close opposite flank.

To describe accurately the wave generation, the granular nature of the falling mass needs to be taken into account $[2,3]$. In the present work, new experiments on the collapse of granular columns into water are presented following the pioneering work of Robbe-Saule et al. [4]. The collapse of dry granular columns has been extensively studied both experimentally $[5,6]$ and numerically [7-10]. The collapse of granular columns totally immersed in water has also been studied recently [11-13]. However, the entry of an initially dry granular column in water and the interplay between the grains and the generated wave remain poorly characterized.

In the present work, a subaerial bidimensional configuration is considered, where many different initial conditions for the column sizes and the initial water depth are investigated. The generated waves display different characteristic nonlinear shapes, connected to the initial geometry of the granular column, the water height and the dynamics of the granular collapse.

\section{Experimental setup}

Waves induced by granular collapses are experimentally reproduced using a setup described in detail in [14]. In summary, a rectangular tank of length $L=2 \mathrm{~m}$ and transverse width $W=0.15 \mathrm{~m}$ contains a water layer of depth $h_{0}$, except at the left-hand side where a granular column of height $H_{0}$ and width $L_{0}$ stands on a solid step of height $h_{0}$

\footnotetext{
*e-mail: wladimir.sarlin1 @ universite-paris-saclay.fr

**e-mail: cyprien.morize@universite-paris-saclay.f
}

A video is available at https://doi.org/10.48448/qsmj-sk52

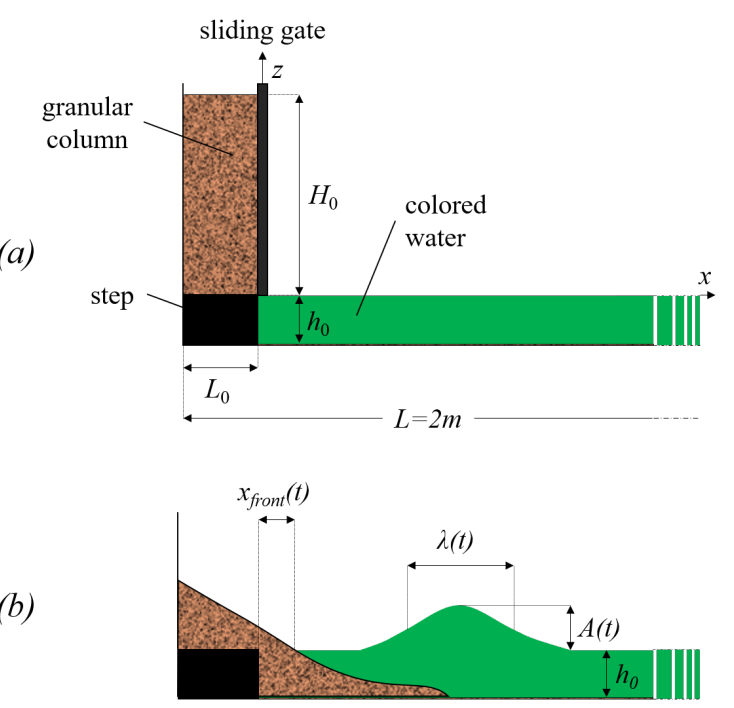

Figure 1. Sketch of the experimental setup : (a) initial subaerial configuration for the granular column and the water tank, and (b) parameters of interest, for both the granular collapse and the generated water wave.

and is initially retained by a vertical sliding gate [Fig. 1]. Quickly lifting this gate using a linear motor operating at a high vertical velocity $(1 \mathrm{~m} / \mathrm{s})$ releases the granular column, which collapses and impacts the water free-surface, leading to the wave generation as illustrated in Fig. 1(b). The time evolution of the collapse and the wave is recorded with a camera, with water colored with fluorescein to enhance the contrast between water, air, and grains [15]. Snapshots from experiments with different initial conditions are presented in Fig. 2(a)-(c). The granular medium consists of glass beads of diameter $5 \mathrm{~mm}$ and density 2500 $\mathrm{kg} \cdot \mathrm{m}^{-3}$. Note that the waves generated would not be very different using other grain diameter and density [14]. The 
aspect ratio of the column, $a=H_{0} / L_{0}$, is varied in the range $0.9 \leqslant a \leqslant 7.8$ and the volume, $V_{0}=H_{0} L_{0} W$, in the range $1.35 \mathrm{dm}^{3} \leqslant V_{0} \leqslant 11.7 \mathrm{dm}^{3}$. Finally, the water height is chosen in the range $2 \mathrm{~cm} \leqslant h_{0} \leqslant 25 \mathrm{~cm}$. Varying these different parameters allows us to investigate the wave generation process in a large range of global Froude number $\mathrm{Fr}_{0}=\sqrt{H_{0} / h_{0}}$, between 0.5 and 4.4. This dimensionless number compares the typical vertical free-fall velocity of the grains $\sqrt{g H_{0}}$ to the classical velocity of linear gravity waves in shallow water $\sqrt{g h_{0}}$. Note that in contrast to the notations used in [14], the height of the column $H_{0}$ is taken here from the top of the column down to the water surface at $z=0$ (top of the solid step). The present experimental work significantly extends the range of global Froude numbers investigated in [14].

Image processing allows us to extract the water freesurface as well as the dry and wet granular profiles. We determine at each time $t$ the amplitude $A(t)$ and the midheight width $\lambda(t)$ of the wave, as well as the position of the front, $x_{\text {front }}(t)$, of the granular collapse at the initial water surface $z=0$. As reported in $[4,14,16]$, the wave grows in amplitude up to a maximum value $A_{m}$ with a corresponding mid-height width $\lambda_{m}$, as illustrated in Fig. 2(b). The wave generation is consecutive to the increase of the immersed volume of grains [16] characterized by a granular front $x_{\text {front }}(t)$ advancing at the local horizontal velocity $v_{\text {front }}=\mathrm{d} x_{\text {front }} / \mathrm{d} t$ [14]. We can thus define a local Froude number $\operatorname{Fr}_{f}=v_{f} / \sqrt{g h_{0}}$ as the ratio of the maximum velocity $v_{f}$ reached by $v_{\text {front }}$ to the velocity of linear water waves [14].
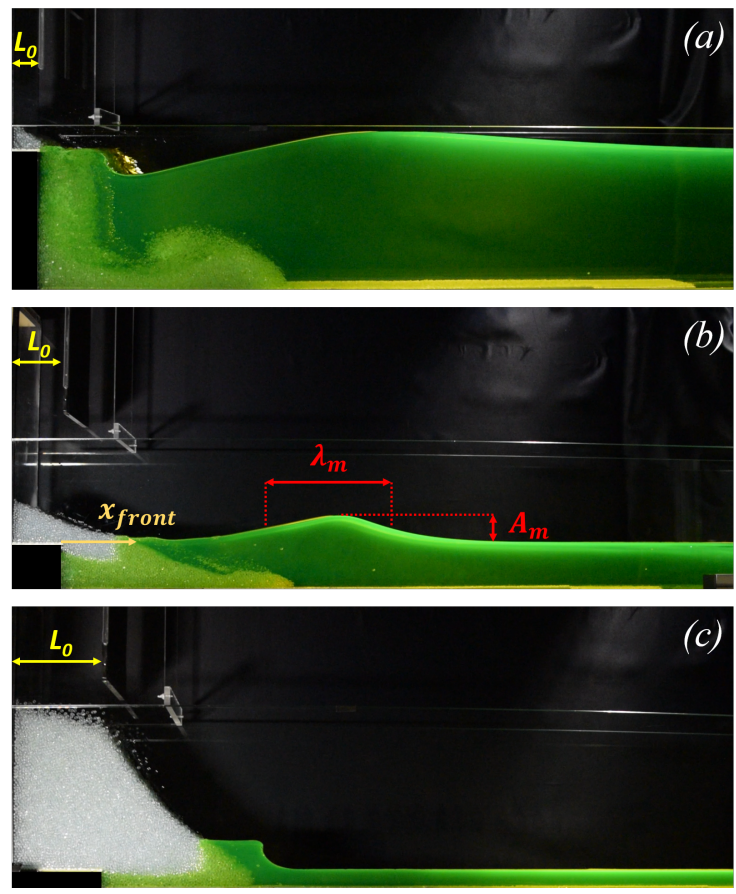

Figure 2. Pictures of the three typical wave shapes observed for different initial conditions : (a) nonlinear transition wave $\left(H_{0}=\right.$ $\left.39 \mathrm{~cm}, L_{0}=5 \mathrm{~cm}, h_{0}=25 \mathrm{~cm}\right)$, (b) solitary wave $\left(H_{0}=29 \mathrm{~cm}\right.$, $\left.L_{0}=10 \mathrm{~cm}, h_{0}=9 \mathrm{~cm}\right)$, and (c) hydraulic jump $\left(H_{0}=39 \mathrm{~cm}, L_{0}\right.$ $\left.=14.5 \mathrm{~cm}, h_{0}=3 \mathrm{~cm}\right)$.

\section{Experimental results}

For a given initial geometry of the granular column, three distinct wave regimes are observed when varying the initial water depth $h_{0}$ and, consequently, the Froude numbers. For small values of the Froude numbers (regime I), a long primary wave followed by a weak dispersive wave train is observed (Fig. 2(a) for $\mathrm{Fr}_{0}=1.25$ and $\mathrm{Fr}_{f}=0.19$ ). This regime corresponds to the nonlinear transition waves reported in [1,17], even though the experimental setups used in these studies are slightly different. At moderate Froude numbers (regime II), quasi-symmetrical solitary waves are observed (Fig. 2(b) for $\mathrm{Fr}_{0}=1.80$ and $\mathrm{Fr}_{f}=0.52$ ), which can either break or not depending on the value of $A_{m} / h_{0}$ reached by the wave, relative to the critical value $A_{m} / h_{0} \simeq 0.73$. Finally, for large Froude numbers (regime III), waves are close in shape to hydraulic jumps, breaking near the collapse region, due to the shock produced at the interface between the grains and the water (Fig. 2(c) for $\mathrm{Fr}_{0}=3.61$ and $\left.\mathrm{Fr}_{f}=1.86\right)$.
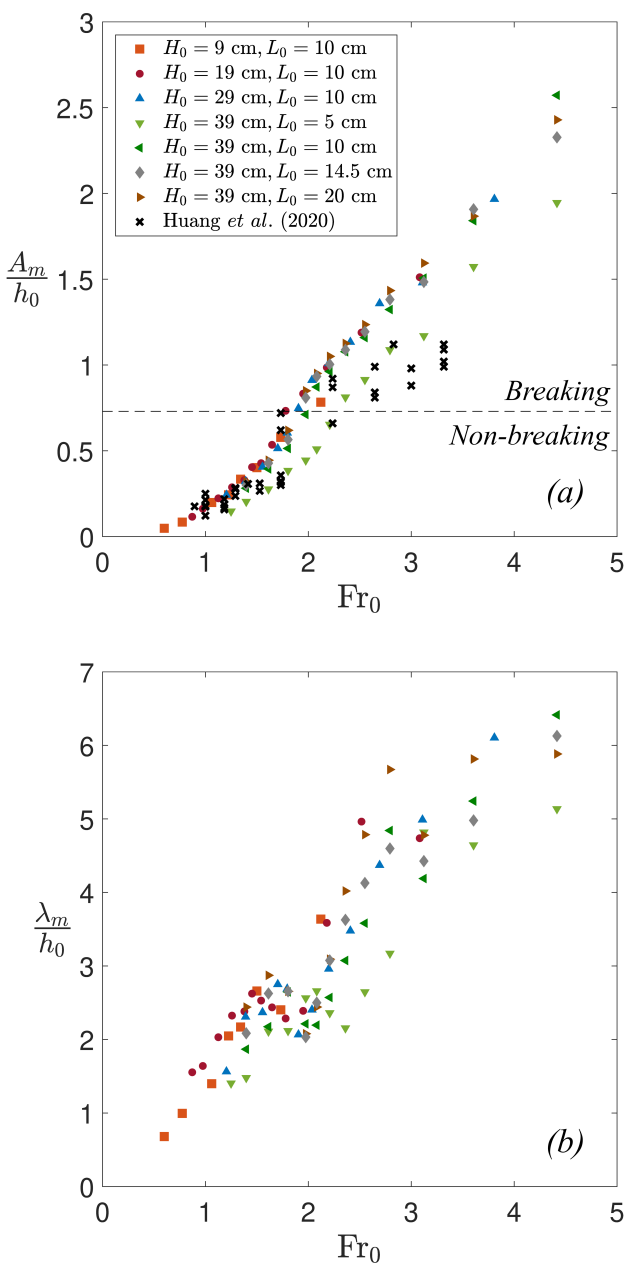

Figure 3. Evolution of (a) the relative wave amplitude $A_{m} / h_{0}$ and (b) the relative mid-height width $\lambda_{m} / h_{0}$ with the global Froude number $\mathrm{Fr}_{0}$, for all our experiments (solid symbols), and the ones of [18] (x); The horizontal dashed line corresponds to the observed transition between breaking and non-breaking waves at $A_{m} / h_{0} \simeq 0.73$. 

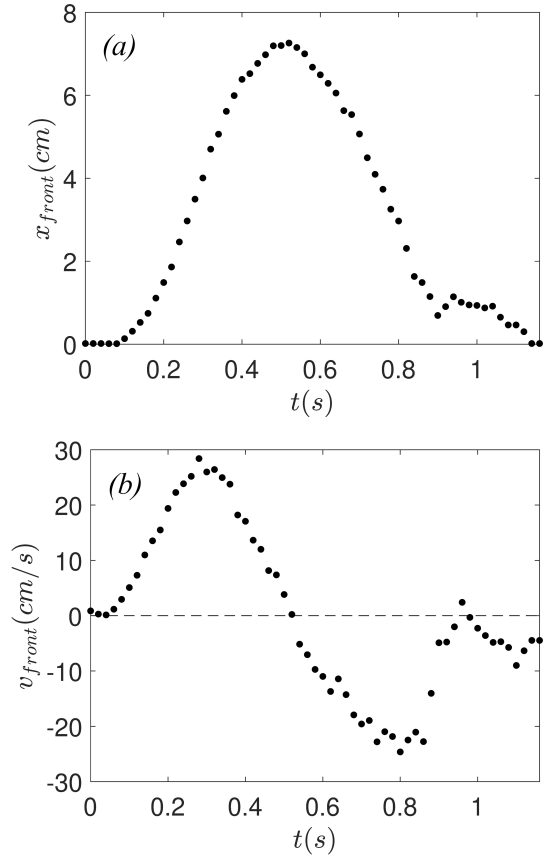

Figure 4. Time evolution of (a) the position, $x_{\text {front }}(t)$, and (b) the velocity $v_{\text {front }}=\mathrm{d} x_{\text {front }} / \mathrm{d} t$ of the granular front for $H_{0}=19$ $\mathrm{cm}, L_{0}=10 \mathrm{~cm}$ and $h_{0}=25 \mathrm{~cm}$ (regime I, for $\mathrm{Fr}_{0}=0.87$ and $\left.\mathrm{Fr}_{f}=0.18\right)$.

The maximum amplitude of the generated wave normalized by the water depth $A_{m} / h_{0}$ and its corresponding relative mid-height width $\lambda_{m} / h_{0}$ are reported in Fig. 3 as a function of the global Froude number $\mathrm{Fr}_{0}$, for all experiments with columns of different sizes $\left(H_{0}, L_{0}\right)$ and different water depths $h_{0}$. A global increasing trend is observed in Fig. 3(a) for $A_{m} / h_{0}$, however the scattering of the data shows that $\mathrm{Fr}_{0}$ is not the sole parameter which governs the wave generation. The aspect ratio of the column also plays a role in the wave generation by modifying the collapse dynamics, as already known for the pure dry case [6]. Note that the data obtained by Huang et al. [18] for larger and non-spherical grains (limestone pebbles of diameter $d \simeq 13 \mathrm{~mm}$ ) in experiments performed at larger-scale $\left(L=16 \mathrm{~m}, W=0.3 \mathrm{~m}, 30 \leqslant H_{0} \leqslant 110 \mathrm{~cm}\right.$, $10 \leqslant h_{0} \leqslant 50 \mathrm{~cm}$ ) follow the same trend. Despite some scattering of our data for the relative mid-height width of the generated waves $\lambda_{m} / h_{0}$, plotted in Fig. 3(b), we observe a departure from a monotonic growth of $\lambda_{m} / h_{0}$ with $\mathrm{Fr}_{0}$ : for $1.5 \lesssim \mathrm{Fr}_{0} \lesssim 2.0$, a slight decrease can be seen, which further supports the experimental observation of three regimes of waves.

The three regimes observed for the generated wave can be related to the different dynamics of the granular collapse in the water. Examples of the time evolution of the granular front $x_{\text {front }}(t)$ at the water surface is shown in Fig. 4(a) and Fig. 5(a) for regimes I and III, respectively. In regime I, corresponding to nonlinear transition waves, $x_{\text {front }}$ first increases to a maximal value $x_{f}$ before decreasing to a final value $x_{f_{\infty}}$ such as $x_{f_{\infty}} \ll x_{f} \ll h_{0}$ [Fig. 4(a)]. In regime III, corresponding to hydraulic jumps, $x_{\text {front }}$ in-
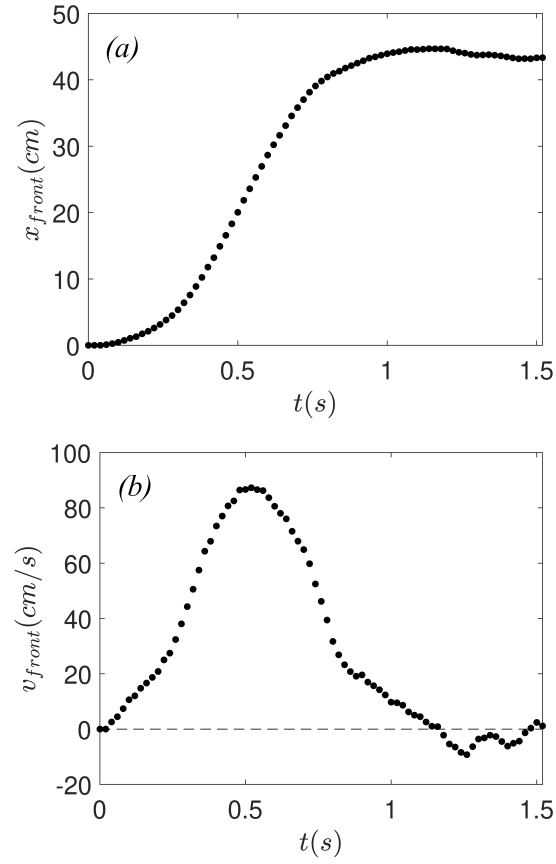

Figure 5. Time evolution of (a) the position, $x_{\text {front }}(t)$, and (b) the velocity $v_{\text {front }}=\mathrm{d} x_{\text {front }} / \mathrm{d} t$ of the granular front for $H_{0}=39$ $\mathrm{cm}, L_{0}=14.5 \mathrm{~cm}$ and $h_{0}=4 \mathrm{~cm}$ (regime III, for $\mathrm{Fr}_{0}=3.12$ and $\mathrm{Fr}_{f}=1.39$ ).

creases continuously from zero to a final value $x_{f_{\infty}} \gg h_{0}$ [Fig. 5(a)]. The time evolution of the horizontal velocity $v_{\text {front }}=\mathrm{d} x_{\text {front }} / \mathrm{d} t$ associated with the granular front is reported in Fig. 4(b) and Fig. 5(b), respectively. For regime $\mathrm{I}, v_{\text {front }}(t)$ exhibits a positive maximum $v_{f}$, but also a negative minimum at the end stage of the collapse, corresponding to the receding phase of the granular front before stop. In regime III, the velocity of the front exhibits a bell shape, from zero up to a maximal value $v_{f}$.

We now report the evolution of $A_{m} / h_{0}$ and $\lambda_{m} / h_{0}$ as a function of the local Froude number $\mathrm{Fr}_{f}=v_{f} / \sqrt{g h_{0}}$ in Fig. 6(a) and (b), respectively. All the data for the relative wave amplitude $A_{m} / h_{0}$ collapse onto a master curve with an increasing continuous trend. The corresponding relative wave width $\lambda_{m} / h_{0}$ displays a more complicated evolution, with a clear decreasing trend characteristic of regime II.

In regime I, corresponding to nonlinear transition waves, $A_{m} / h_{0}$ and $\lambda_{m} / h_{0}$ both strongly increase with $\mathrm{Fr}_{f}$ up to $A_{m} / h_{0} \simeq 0.35$ and $\lambda_{m} / h_{0} \simeq 2.8$ for $\mathrm{Fr}_{f} \lesssim 0.35$. In regime II, corresponding to nearly symmetrical solitary waves, $A_{m} / h_{0}$ increases up to $A_{m} / h_{0} \simeq 1.0$ but $\lambda_{m} / h_{0}$ now slightly decreases from 2.8 to 2.1 as $\operatorname{Fr}_{f}$ increases from 0.35 to 0.87 . In regime III, corresponding to hydraulic jumps, $A_{m} / h_{0}$ and $\lambda_{m} / h_{0}$ again both increase with $\mathrm{Fr}_{f}$ but with a large scattering for $\lambda_{m} / h_{0}$. For this regime, $\lambda_{m}$ is also related with the front extension $x_{f}$.

\section{Conclusions}

In this study, we report that tsunami waves generated by the collapse of a dry granular column into water 

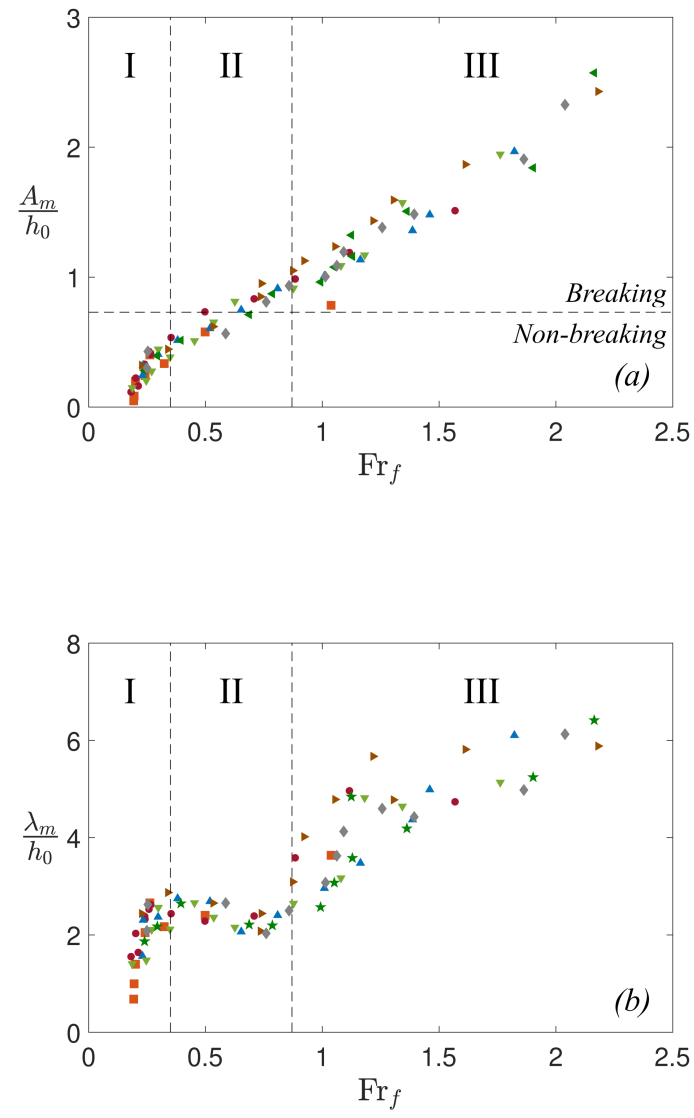

Figure 6. Evolution of (a) the relative amplitude $A_{m} / h_{0}$ and (b) the relative mid-height width $\lambda_{m} / h_{0}$ of the wave with the local Froude number $\mathrm{Fr}_{f}$ for all our experiments (same data symbols as in Fig. 3). The two vertical dashed lines correspond to the transitions at $\mathrm{Fr}_{f}=0.35$ between nonlinear transition waves (I) and solitary waves (II), and at $\mathrm{Fr}_{f}=0.87$ between solitary waves (II) and hydraulic jumps (III).

display different characteristic shapes, governed by a local Froude number $\mathrm{Fr}_{f}$ based on the horizontal velocity of the granular front at the water free-surface. Up to $\mathrm{Fr}_{f} \simeq 0.35$, nonlinear transition waves are generated with increasing relative amplitude and relative mid-height width with $\mathrm{Fr}_{f}$. For $0.35 \lesssim \mathrm{Fr}_{f} \lesssim 0.87$, the relative amplitude of the solitary waves increases with $\mathrm{Fr}_{f}$, while the relative width decreases. Finally, for $\mathrm{Fr}_{f} \gtrsim 0.87$, hydraulic jumps are observed while both the relative amplitude and relative width increase with $\mathrm{Fr}_{f}$. Based on this local Froude number, a clear separation between these three regimes is possible. The experiments reported here allow to characterize the hydrodynamics of landslide generated waves for a range of initial conditions that cover most of the real cases observed in Nature [16], from very shallow water to deep water scenarios. The accurate hydrodynamic modeling of these three regimes is in progress.

We are grateful to J. Amarni, A. Aubertin, L. Auffray and R. Pidoux for the development of the experimental setup, and to E. Lajeunesse and P.-Y. Lagrée for fruitful discussions.

\section{References}

[1] H. Fritz, W. Hager, H. Minor, Sci. Tsunami Haz. 19, 3-19 (2001)

[2] H. Fritz, W. Hager, H. Minor, J. Waterway Port Coast. Ocean Eng. 130, 287-302 (2004)

[3] S. Viroulet, A. Sauret, O. Kimmoun, EPL 105, (2014)

[4] M. Robbe-Saule, C. Morize, Y. Bertho, A. Sauret, P. Gondret, EPJ Web of Conf. 140, 14007 (2017)

[5] N.J. Balmforth, R.R. Kerswell, J. Fluid Mech. 538, 399-428 (2005)

[6] E. Lajeunesse, J.B. Monnier, G.M. Homsy, Phys. Fluids 17, 103302 (2005)

[7] R. Zenit, Phys. Fluids 17, 031703 (2005)

[8] E. Larrieu, L. Staron, E.J. Hinch, J. Fluid Mech. 554, 259-270 (2006)

[9] L. Lacaze, J.C. Phillips, R.R. Kerswell, Phys. Fluids 20, 063302 (2008)

[10] P.-Y. Lagrée, L. Staron, S. Popinet, J. Fluid Mech. 686, 378-408 (2011)

[11] L. Rondon, O. Pouliquen, P. Aussillous, Phys. Fluids, 23, 073301 (2011)

[12] V. Topin, Y. Monerie, F. Perales, F. Radjai, Phys. Rev. Lett. 109, 188001 (2012)

[13] A. Bougouin, L. Lacaze, Phys. Rev. Fluids 3, 064305 (2018)

[14] M. Robbe-Saule, C. Morize, R. Henaff, Y. Bertho, A. Sauret, P. Gondret, J. Fluid Mech. 907, A11 (2021)

[15] http://www.fast.u-psud.fr/galerie/tsunami/tsunami. php

[16] M. Robbe-Saule, C. Morize, Y. Bertho, A. Sauret, A. Hildenbrand, P. Gondret, submitted to Geophys. Res. Lett.

[17] S. Viroulet, D. Cébron, O. Kimmoun, C. Kharif, Geophys. J. Int., 193, 747-762 (2013)

[18] B. Huang, Q. Zhang, J. Wang, C. Luo, X. Chen, L. Chen, Phys. Fluids 32, 033301 (2020) 